Original article

\title{
Comparative experimental assessment of the accuracy of nanosecond laser surgery of the oral cavity when the instrument is moved by a robotic complex and a surgeon
}

\author{
Andrey A. Chunikhin ${ }^{1}$, Ernest A. Bazikyan ${ }^{1}$, Yury V. Poduraev ${ }^{2}$, Andrey A. Vorotnikov ${ }^{2}$, Daniil D. Klimov ${ }^{2}$ \\ ${ }^{1}$ Moscow State University of Medicine and Dentistry n.a. A.I. Evdokimov, Moscow, Russia \\ ${ }^{2}$ Stankin Moscow State Technological University, Moscow, Russia
}

Received 12 May 2018, Revised 23 August 2018, Accepted 19 December 2018

(C) 2018, Chunikhin A.A., Bazikyan E.A., Poduraev Yu.V., Vorotnikov A.A., Klimov D.D.

(C) 2018, Russian Open Medical Journal

Abstract: Aim - to compare the constancy of maintaining the size of the air gap in standard surgical incisions of the oral mucosa using a diode laser performed by a manipulation robot and by the hand of a surgeon.

Material and Methods - A manipulative robotic mechanism coupled with a medical instrument - a semiconductor laser with a nanosecond pulsed pumping driver with a wavelength of $\lambda=1260 \mu \mathrm{m}$ was used for the study. A comparative tool for simulating manual movement was a working organ with a nozzle for holding in surgeon's hand. The study was conducted on three typical trajectories: linear, semilunar and scalloped. The point coordinates were obtained by scanning the position of the reflector of the coordinate measuring machine in 100 passes with the calculation of the value of the standard deviation of the various measured quantities.

Results - it was estimated that the magnitude of the spread of the air gap on typical trajectories between the laser tip and the treated biological tissue perfofmed by the robot is on the average 7 times less $(p<0.05)$ compared to manual movements.

Conclusion - The results showed that the use of robotic laser complexes in medicine would significantly improve the accuracy of the medical laser movement maintaining a constant fixed distance between the laser instrument and the mucosa in a range of up to $1 \mathrm{~mm}$.

Keywords: biomedical laser technology, laser robotic surgery, nanosecond pulsed laser, coordinate measuring machines

Cite as Chunikhin AA, Bazikyan EA, Poduraev YuV, Vorotnikov AA, Klimov DD. Comparative experimental assessment of the accuracy of nanosecond laser surgery of the oral cavity when the instrument is moved by a robotic complex and a surgeon. Russian Open Medical Journal $2019 ; 8$ : e0307.

Correspondence to Andrey A. Chunikhin. Address: Department of oral surgery, Moscow State University of Medicine and Dentistry named after A.I. Evdokimov, 20/1 Delegatskaya St., Moscow, 127473, Russia. E-mail: docca74@yandex.ru.

\section{Introduction}

The development of robotic complexes in various branches of industrial production in recent decades has not left aside medicine. Surgical robotic systems began their development in medical practice with the positioning of endoscopic instruments, allowing to help the surgeon to control the video image of the surgical field and the accuracy of the instrument direction in any laparoscopic surgery [1]. Such systems began to be used in 1995 in performing operations of hip joint replacement based on computed tomography images [2].

The success of many robotic operations carried out around the world, including in Russia, using the daVinci robotic surgical system, testifies to the widespread adoption of robotic technologies in various fields of medicine. The experience of using this system shows significant advantages of robotized technologies over laparoscopic techniques and even more so over open abdominal interventions conducted by a surgeon [1, 3].

The results of the development of industrial robotics are reflected in mechanical design, kinematics, control algorithms and programming. Researchers in [4] actively improve the approaches associated with improving the accuracy of positioning of the robots. Currently, these developers are also working on such parameters of robots as adaptability, in which the use of sensory information makes it possible to respond to changing conditions, and autonomy - the ability to perform tasks without human observation.

Laser photoablation has been used in medicine since the development of the laser in 1960, and its first medical use was registered in 1961 in ophthalmology [5].

The use of various laser systems in dentistry is widely spread. Surgical treatment in the head and neck is associated with incisions, tissue release, rapid rehabilitation, minimization of invasion. Due to the complex three-dimensional anatomy and the close proximity of the thinnest anatomical structures, the intervention requires the highest precision. This approach is difficult, since the oral cavity is one of the most common access routes in surgical dentistry, and damage to the thinnest anatomical structures: muscles, vessels, nerves and pneumatic sinuses can lead to serious complications, increasing the duration of treatment [6].

Laser radiation in the wavelength range of $630-1300 \mu \mathrm{m}$ promotes the manifestation of stimulation of electrolyte metabolism in the protoplasm of cells, accelerates metabolism, increases cell proliferative activity, has fibrinolytic and thrombolytic properties and anti-inflammatory and anti-edema effects [7]. 


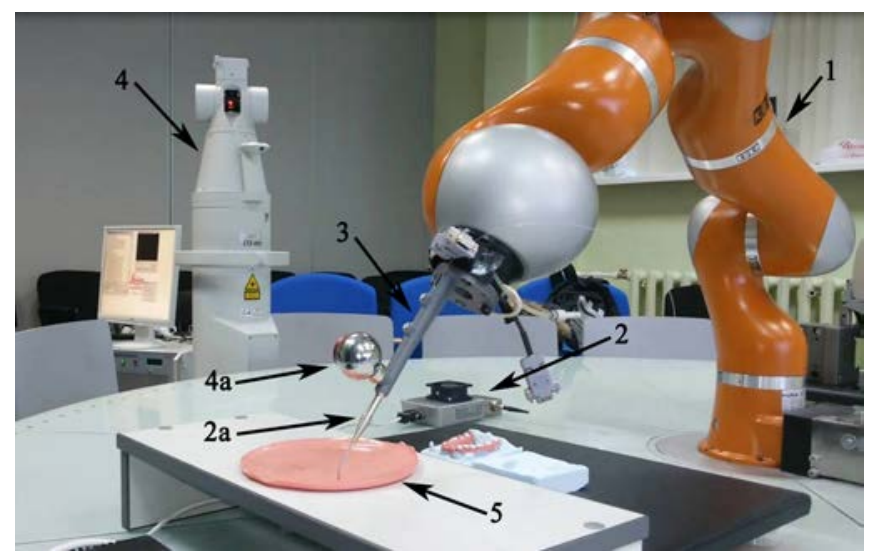

Figure 1. Appearance of the manipulation robot for maxillofacial surgery: 1- LBR 4+ manipulator 2 - Nanosecond pulsed laser module: 2a - holder of a laser light guide; 3 - a working tool connecting the handling robot with the laser module; 4 -Coordinate measuring machine: $4 a-$ reflector of the coordinate measuring machine; 5 - artificial mucosa.

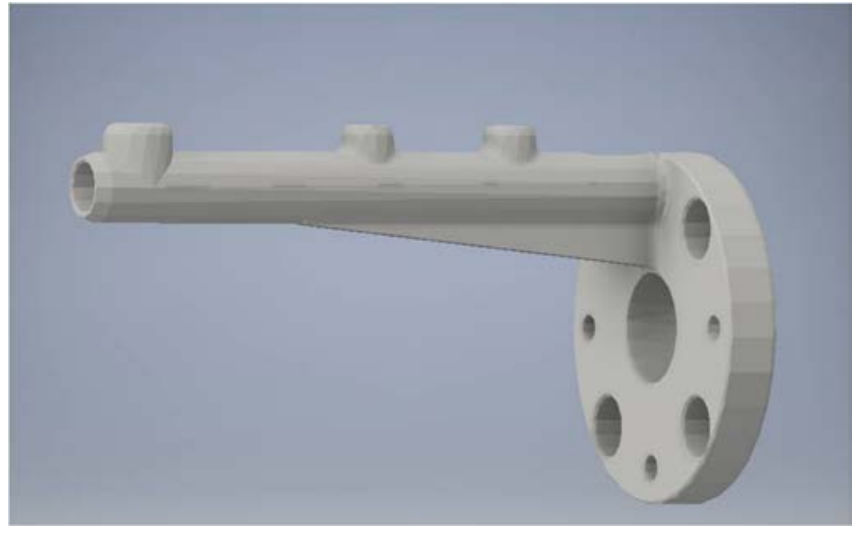

Figure 2. 3D model of the working body.

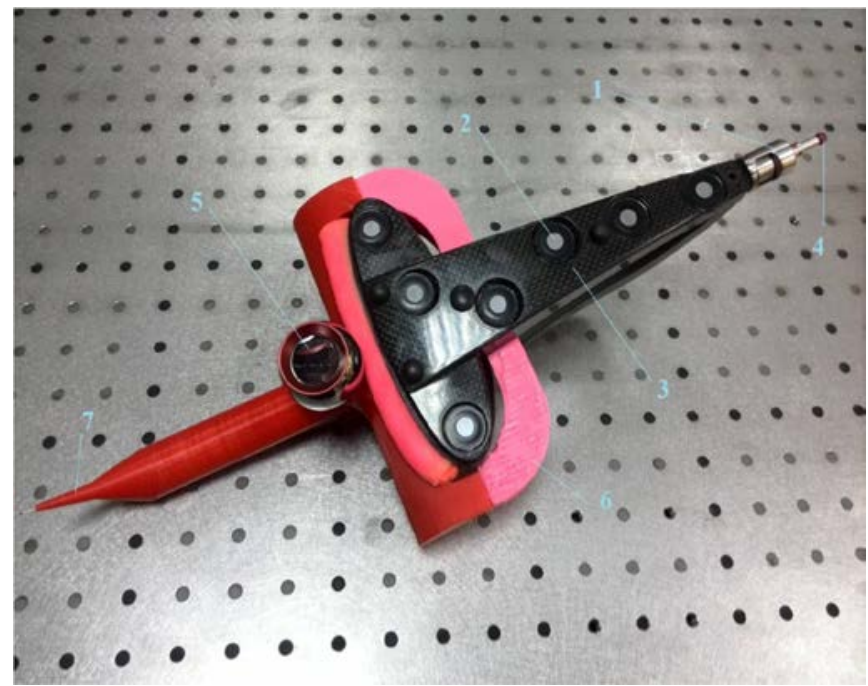

Figure 3. The working body for measuring the position and speed during manual movement: 1 - the probe of a stereophotogrammetric coordinate measuring machine; 2 - reflector; 3 - hard base; 4 - a spherical ruby tip of a stereo gram-metric machine; 5 - reflector of the coordinate measuring system; 6 - connecting equipment; 7 -the nozzle imitating the medical tool.
The development of laser technology has led to the development of new devices with unique laser radiation characteristics, compact dimensions and the possibility of combined exposure of low-intensity and high-intensity radiation, using photochemical effects on oxygen in tissues, which allows to perform non-contact laser surgery of the soft tissues of the maxillofacial region with minimal mechanical and thermal effects on bone and surrounding tissues $[8,9]$.

However, the use of laser technology in a robotic system would increase the precision of the laser instrument, which, in turn, would allow to perform more accurate and minimally invasive surgical interventions compared with the surgeon.

Development and creation of a robotic surgical system for the treatment of complex pathology of the maxillofacial area with the ability to integrate the laser module into the robot arm as a working tool is a promising direction for the development of the medical industry and the implementation of a program of improving medical care in Russia.

A group of researchers, dentists from Moscow State University of Medicine and Dentistry n.a. A.I. Evdokimov (Moscow, Russia), together with robotics engineers at Stankin Moscow State Technological University (Moscow, Russia), developed a robotic multifunctional surgical complex for the maxillofacial area based on a manipulation robot that allows to evaluate the robotic laser capabilities in surgery of the maxillofacial area in terms of accuracy and minimal invasion compared to manual use of laser surgical technology.

The purpose of this study is to assess the error in the size of the air gap between the laser tip and the biological tissue - the difference between the measured and the specified air gap value while performing standard surgical incisions using laser radiation tip inbuilt in the manipulation robot compared to the same manipulations by the hand of a surgeon.

\section{Material and Methods}

\section{Experimental setup for measuring robot movements}

The assembly of an experimental stand of a multifunctional surgical complex for laser therapy and surgery of tissues of the maxillofacial area is shown in Figure 1.

The robotic stand system includes a manipulation mechanism LBR 4+ made by KUKA (Germany), which is a seven-part articulated mechanism with a consistent kinematic structure (1).

The medical instrument in the handling robot is a laser module (2), the optical radiation of which is created by a multimode semiconductor laser based on the Ga-A-Al structure with direct current pumping. The module is a nanosecond pulsed pump driver with a wavelength of $\lambda=1260 \mu \mathrm{m}$. The main characteristics of the laser working tool are shown in Table 1.

An optical fiber with a diameter of $400 \mu \mathrm{m}$, covered with a protective plastic sheath, emerges from the medical instrument and enters the holder of the optical fiber (2a), which in turn is fixed in the working instrument (3) connecting it to the flange of the handling robot. The impact of laser radiation on biological tissue occurs directly from the distal end of the fiber.

To control the operating modes of the laser installed on the robot, the channels of the laser power supply are connected via electromagnetic relays to the discrete output unit of the robot control device. 
Table 1. The main optical characteristics of the laser working too

\begin{tabular}{|c|c|c|c|c|}
\hline \multirow[t]{2}{*}{ Parameter name } & \multicolumn{3}{|c|}{ Value } & \multirow{2}{*}{$\begin{array}{c}\text { Unit of } \\
\text { measurement }\end{array}$} \\
\hline & Min. & Nom. & Max. & \\
\hline Optical wavelength & 1256 & 1260 & 1266 & $\mu \mathrm{m}$ \\
\hline $\begin{array}{l}\text { Numerical aperture of } \\
\text { optical fiber }\end{array}$ & & 0,22 & & - \\
\hline Operation mode & \multicolumn{4}{|c|}{ Pulse (meander) } \\
\hline Optical pulse duration & 50 & 50 & 50 & ns \\
\hline Optical pulse repetition rate & 0,5 & 0,5 & 0,5 & $\mathrm{MHz}$ \\
\hline Current consumption & - & 2,5 & 2,5 & A \\
\hline Current consumption & - & 2,5 & 2,5 & $A$ \\
\hline Power consumption & - & 100 & - & W \\
\hline $\begin{array}{l}\text { Operating ambient } \\
\text { temperature }\end{array}$ & 18 & 25 & 35 & ${ }^{\circ} \mathrm{C}$ \\
\hline $\begin{array}{l}\text { The shift of the radiation } \\
\text { wavelength depending on } \\
\text { temperature }\end{array}$ & 2,8 & 3,0 & 3,2 & $\AA ̊ \cap /{ }^{\circ} \mathrm{C}$ \\
\hline Continuous working time & 1 & 1 & 1 & hour \\
\hline
\end{tabular}

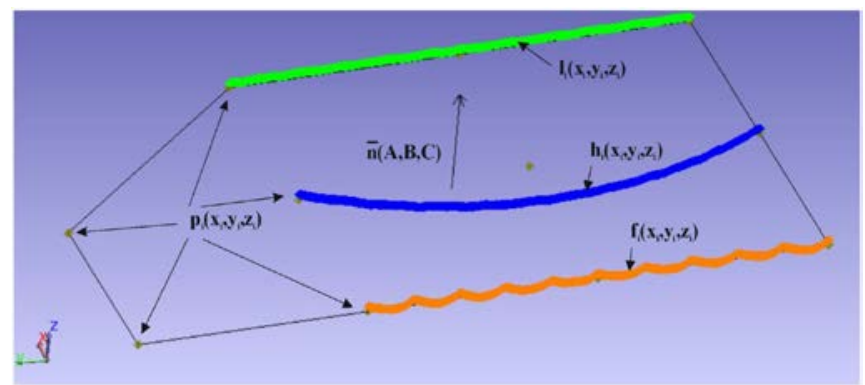

Figure 4. A set of coordinates of the position of the medical instrument on the linear $-\boldsymbol{l}_{i}\left(\boldsymbol{x}_{\boldsymbol{i}}, \boldsymbol{y}_{\boldsymbol{i}} \mathbf{z}_{i}\right)$, semilunar $-\boldsymbol{h}_{i}\left(\boldsymbol{x}_{\boldsymbol{i}}, \boldsymbol{y}_{i}, z_{i}\right)$ and scalloped $-f_{i}\left(x_{i}, y_{i}, z_{i}\right)$ trajectories.

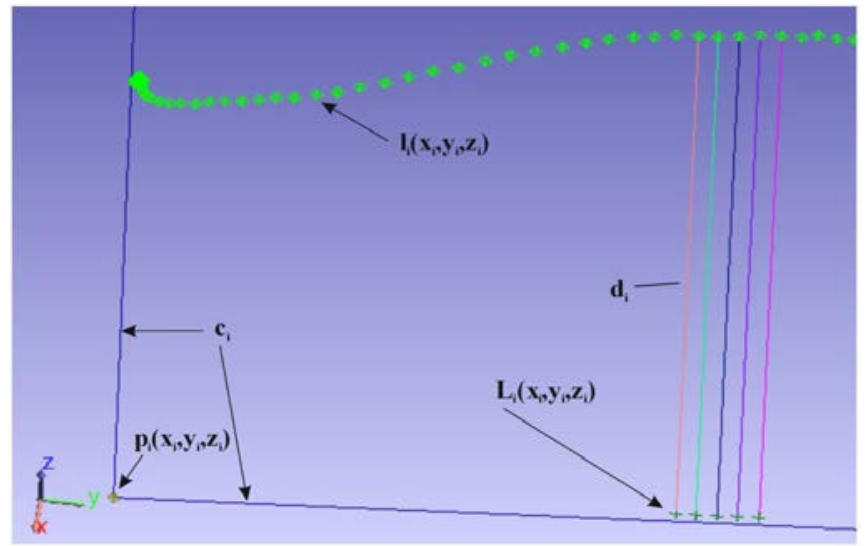

Figure 5. Determining the distance $d_{i}$ from every point $I_{i}\left(x_{i}, y_{i}, z_{i}\right)$ to its projection $L_{i}\left(x_{i}, y_{i}, z_{i}\right)$ on a flat plane, relative to which it was required to maintain an air gap.

The LBR 4+ controller uses a 16 channel discrete output module BECKHOFF KL2809.

To switch the channels of the laser power supply unit by the control signal from the robot controller, an electromagnetic relay is used. This relay controls the $+24 \mathrm{~V}$ signal supplied from the digital output unit.

The working tool (3) is designed to interface the holder of the optical fiber of a medical laser with a 6-power manipulator flange, as well as to fix the laser reflector of the coordinate measuring machine (4a) on it, which is necessary to control the position and speed of the working tool while it moves along the path.

For the manufacture of the prototype of the working body, a sketch drawing was made and a 3D model of the working body was created (Figure 2).

The printing of the developed working tool model was carried out on a 3D printer using heat-resistant plastic.

The Leica Laser Tracker LTD8 coordinate measuring machine from Leica (Germany) was used to measure coordinate points during functional movements and typical motion paths (4).

For the experiment, artificial gums were made to simulate the incision using laser radiation in minimally invasive optical surgical equipment (5), whose composition is a $5-25 \%$ aqueous solution of gelatin with added aminoacetic acid or proline, taken in an amount of 8-10 wt. \% to the total amount of solution. This technical solution is used because of the fact that for the radiation of a diode laser with an operating wavelength range of $1250-1270 \mu \mathrm{m}$ the maximum absorption with the release of singlet oxygen is observed [10].

\section{Experimental setup for measuring manual movements}

To determine the position and orientation of the medical instrument during its manual movement, a working body has been developed, shown in Figure 3.

\section{Statistical analysis}

The standard deviation of the various measured values is calculated in accordance with the following expression:

$$
\sigma=\sqrt{\frac{1}{n} \cdot \sum_{i=1}^{n}\left(p_{i}-p_{\text {mid }}\right)^{2}}
$$

where, $\Sigma-$ the average value of the measured value, $p_{\text {mid }}-$ the value of the measured value at each point $p_{i}, n-$ the number of points.

The studies were conducted along the following typical movement paths of a medical instrument, which are shown in Figure 4: linear, semilunar, and scalloped. Measurements of the coordinates of points of typical trajectories are carried out using a coordinate measuring machine. The values of the coordinates of the points are obtained by continuously scanning the position of the reflector mounted on the magnetic base of the working tool, or on the working tool of a surgical robotic laser system. Derived on typical motion paths (linear $l_{i}\left(x_{i}, y_{i}, z_{i}\right)$, lunate $h_{i}\left(x_{i}, y_{i}, z_{i}\right)$ and scalloped $\left.f_{i}\left(x_{i}, y_{i}, z_{i}\right)\right)$ coordinates of the position of the medical instrument are shown in Figure 4, where $i$ - number of measured coordinates of points, $i=1 . . n$, and $n-$ number of scan points.

\section{Results}

For making incisions of the required depth and width, it is necessary to withstand the nominal (specified) air gap $d_{n}$ between the laser tip and the biological tissue. For conducting the experiments comparing the movements of the robot and the manual movements of the surgeon, the flat surface with minimal flatness was used, and for evaluating the movements of the robot, the flat surface is set to the robot using the program. To determine the plane using the reflector of the coordinate measuring machine, 
the coordinates of the points are measured $p_{i}\left(x_{i}, y_{i}, z_{i}\right)$ which are depicted in Figure 5.

To find the parameters of the plane $\left(A^{\prime}, B^{\prime}, D^{\prime}\right)$ all coordinates of the points of the trajectory are approximated by the least squares method by the following expression:

$$
\left(\begin{array}{l}
A^{\prime} \\
B^{\prime} \\
D^{\prime}
\end{array}\right)=\left(\begin{array}{ccc}
\sum_{i=1}^{N} x_{i}{ }^{2} & \sum_{i=1}^{N} x_{i} \cdot y_{i} & \sum_{i=1}^{N} x_{i} \\
\sum_{i=1}^{N} x_{i} \cdot y_{i} & \sum_{i=1}^{N} y_{i}{ }^{2} & \sum_{i=1}^{N} y_{i} \\
\sum_{i=1}^{N} x_{i} & \sum_{i=1}^{N} y_{i} & N
\end{array}\right)^{-1} \cdot\left(\begin{array}{c}
\sum_{i=1}^{N} x_{i} \cdot z_{i} \\
\sum_{i=1}^{N} y_{i} \cdot z_{i} \\
\sum_{i=1}^{N} z_{i}
\end{array}\right)
$$

To go to $(a, b, c)$ expression is used:

$$
\left(\begin{array}{l}
a \\
b \\
c
\end{array}\right)=\left(\begin{array}{c}
A^{\prime} / \mathrm{D}^{\prime} \\
B^{\prime} / \mathrm{D}^{\prime} \\
1 / \mathrm{D}^{\prime}
\end{array}\right)
$$

They are transformed into unit vectors according to the Euclidean norm and the normal vector is determined $\bar{n}(A, B, C)$ to the plane:

$$
\left(\begin{array}{l}
A \\
B \\
C
\end{array}\right)=\cdot\left(\begin{array}{l}
a / \sqrt{a^{2}+b^{2}+c^{2}} \\
b / \sqrt{a^{2}+b^{2}+c^{2}} \\
c / \sqrt{a^{2}+b^{2}+c^{2}}
\end{array}\right)
$$

Distance from the origin to the measured plane $D_{m}$ defined as the arithmetic average of the distances to each measured point:

$$
D_{i}=A \cdot x_{i}+B \cdot y_{i}+C \cdot z_{i}
$$

The value of the air gap for each measured point coordinates is determined in accordance with the following formula:

$$
d_{i}=A \cdot x_{i}+B \cdot y_{i}+C \cdot z_{i}-D_{m}
$$

Where $\left(x_{i}, y_{i}, z_{i}\right)$ these are coordinates linear $-l_{i}\left(x_{i}, y_{i}, z_{i}\right)$, semilunar $-h_{i}\left(x_{i}, y_{i}, z_{i}\right)$ or scalloped $-f_{i}\left(x_{i}, y_{i}, z_{i}\right)$ trajectories of both the robot and the surgeon. Figure 5 shows: the contour of the measured plane $C_{i}$ and air gap $d_{i}$ from each point of the linear trajectory of movement $l_{i}\left(x_{i}, y_{i}, z_{i}\right)$ medical instrument to its projection $l_{i}\left(x_{i}, y_{i}, Z_{i}\right)$ on the plane of the table.

When conducting the experiment, both the surgeon and the robot were required to sustain $1 \mathrm{~mm}$ between each point of any typical trajectory, which are shown in Figure 4, and a flat plane. Before comparison, it is customary to divide the typical trajectories conducted by the surgeon into two categories: these are trajectories, the passage through which was carried out with the presence of a fulcrum, i.e. the surgeon's hand touches the surface of the table during the passage, and without touching the trajectory ("in the air"). This is due to the need for the surgeon to hold the cutting tool in different ways during operations, depending on the openness of the surgical field.
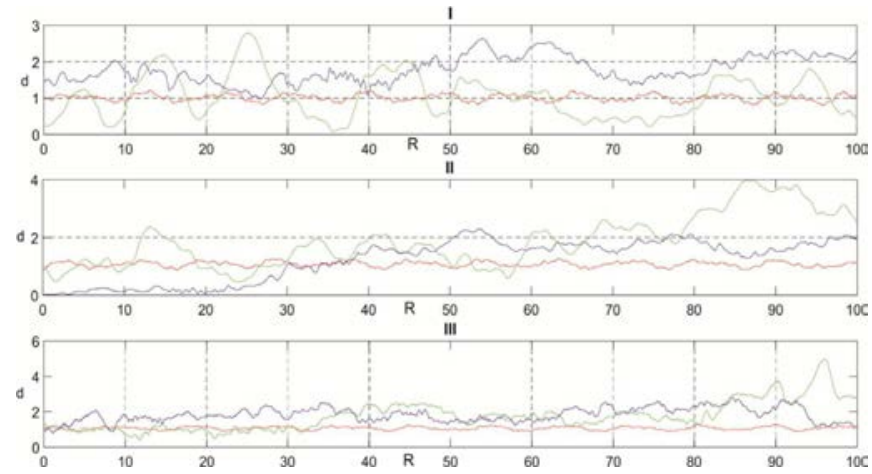

Figure 6. Graphs of changes in the size of the air gap $d_{i}(\mathrm{Mm})$ from the position of a medical instrument $\boldsymbol{R}_{i}$ ( $(\mathrm{MM})$ with manual movements with a touch point (color - blue), without a touch point (color - green) and when moving with a robot (color - red) along typical paths (I - linear, II - semilunar, III scalloped).
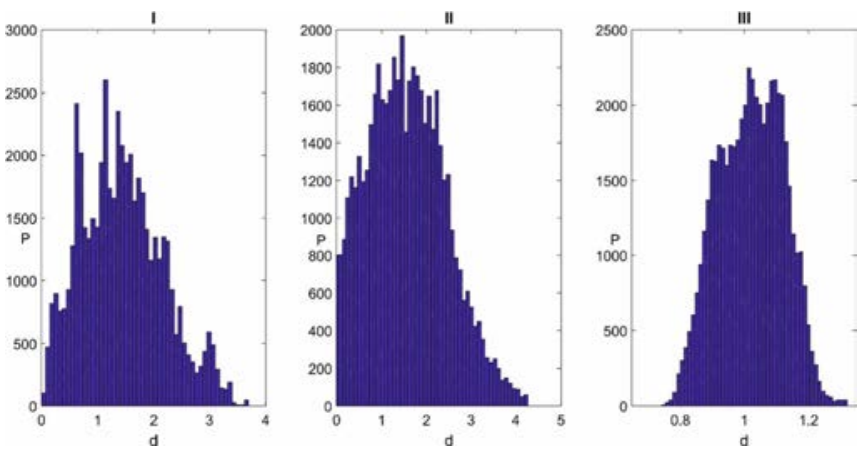

Figure 7. Histograms of the air gap size distribution $d_{i}$ at manual movements with a point of contact (I), without a point of contact (II) and when moving by a robot (III) along all typical trajectories.

Table 2. The error of the size of the air gap on the typical trajectories

\begin{tabular}{lccccc}
\hline Trajectory & $\delta_{p}(\mathrm{~mm})$ & $\delta_{a}(\mathrm{~mm})$ & $\delta_{R}(\mathrm{~mm})$ & $\delta_{p} / \delta_{R}$ & $\delta_{a} / \delta_{R}$ \\
\hline Linear & 0.782 & 0.048 & 0.112 & 7 & 0 \\
Semilunar & 0.217 & 0.862 & 0.075 & 3 & 11 \\
Scalloped & 0.839 & 0.753 & 0.071 & 12 & 11 \\
\hline
\end{tabular}

Graphs illustrating the size of the air gap $d_{i}$ from the position of a medical instrument $R_{i}$ when passing along each trajectory are shown in Figure 6.

Considering the graphs revealed inconstancy of the size of the air gap during manual movements with both a hand fulcrum and no fulcrum compared to a robot. During the scanning of the trajectories of manual movements, it was revealed that the surgeon cannot stop the medical instrument at the exact point of the specified trajectory, but stops there with a position error (1.3 $\mathrm{mm}$ on average), unlike a robot $(0.15 \mathrm{~mm})$. Therefore, when plotting graphs for comparing a surgeon and a robot, there are no data on the end of the trajectory of manual movements. The nominal distance between the first and last point of each type path is $100 \mathrm{~mm}$. For each typical trajectory, scan points consist on average of 3300 points. 
The difference between the average air gap obtained $d_{m}$ and the specified $d_{n}$ is the error of the air gap.

$$
\delta=d_{n}-d_{m}
$$

Average air gap $d_{m}$ on the trajectory is defined as the arithmetic mean $d_{i}$ on every single trajectory.

The values of the error of the size of the air gap for each path during manual movements with a touch point $\left(\delta_{p}\right)$, no touch point $\left(\delta_{a}\right)$ and when moved by robot $\left(\delta_{R}\right)$ presented in Table 2 .

If an $\delta_{R}$ on all typical paths have similar meanings, then $\delta_{p}$ and $\delta_{a}$ significantly differ from each other. In this regard, the evaluation of $\delta_{p} / \delta_{R}$ and $\delta_{a} / \delta_{R}$ ratios is rather ambiguous and nevertheless can be used together with the robot's additional movements compared to the second criterion, since average arithmetic relations $\delta_{p} / \delta_{R}$ and $\delta_{a} / \delta_{R}$ for linear, semilunar and scalloped trajectories are equal to 7 and 7 respectively.

So, the size of the air gap during manual movements is not constant, so it is necessary to conduct statistical experiments to identify an additional criterion, which will depend on the distribution of the size of the air gap.

When carrying out manual movements in five passes in each typical trajectory, there was no significant difference in the nature of the distribution of the air gap size, so it was decided to combine the experimental data on the linear, semilunar and scalloped trajectories into a single data file. Thus, to assess the nature of the distribution of the air gap size, 15 trajectories of all manual movements with a touch point, without a touch point and robot movements, 3 data arrays with a total number of points equal to 51863, 50749 and 53705, respectively, are used. Figure 7 shows the histograms of the distribution of the size of the air gap $d_{i}$ along all typical paths.

According to the obtained data of statistical experiments, the absence of normal distribution was determined in accordance with the Kolmogorov-Smirnov compliance criterion and the Pearson compliance criterion. Therefore, for an additional criterion for comparing the movements of the robot and manual movements, the amount of scatter $\varepsilon$ is taken, the difference between the maximum and minimum air gap values in each sample. The magnitude of the air gap spread over all typical paths for manual movements is: with a tangency point (I) $\varepsilon_{p}=3.693 \mathrm{~mm}$, without touch point (II) $\varepsilon_{a}=4.307 \mathrm{~mm}$ and when moved by a robot (III) $\varepsilon_{R}=0.579 \mathrm{~mm}$. When comparing the values of the air gap of manual movements, it is possible to conclude that the presence of a fulcrum while making movements contributes only to a small decrease in the magnitude of the spread. It was also found that the moment of contact between the tip of a medical instrument and the surface of a flat plane on which trajectories were scanned in the case of manual movements with a touch point occurred about 10 times, and in the case without a point of support about 80 times (provided that one contact averages 10 scan points). In the case of a robot, the moment of contact between the tip of the medical instrument and the table did not come even once. The robot stably holds the size of the air gap and has stiffness sufficient for a large number of diverse operations without the need for an additional fulcrum compared with the surgeon.
It is estimated that the amount of scatter of the air gap on typical paths between the laser tip and the biological tissue moved by a robot is 6 times smaller than the manual movement with a touch point $\left(\varepsilon_{p} / \varepsilon_{R}\right)$. It is also estimated that the amount of scatter of the air gap on typical paths between the laser tip and the biological tissue moved by a robot is 7 times smaller than the manual movement without a touch point $\left(\varepsilon_{a} / \varepsilon_{R}\right)$.

\section{Discussion}

Currently, a large number of robotic medical complexes from various fields of medicine are at the stage of operation and development. Researchers [11] are working to create a STAR robotic system capable of autonomously stitching incisions on soft tissues. The articles $[12,13]$ describe the RONNA robotic complex, developed for neurosurgery, in the brain area. Also, a medical robotic complex (medical robotic cell) for osteotomy was developed by researchers [14].

Modern studies do not pay due attention to the quantitative indicators of the relationship between the quality of operations with the help of a robot and operations performed by a surgeon manually. All of the above complexes [1-3] and many others certainly solve complex medical problems. At the same time, during all operations with their help, the question arises of the quality of treatment. Modern surgical practice associates the quality of treatment with the patient's condition before and after surgery [5-7]. And now, the qualitative indicators of surgical operations with the use of medical lasers are minimal blood loss, antibacterial effect, the amount of time of the postoperative period, etc. [6-7]. However, during operations on the gums, the quality of treatment depends not only on the patient's condition, but also on the quality of the laser tip manipulation. The quality depends primarily on the quality of the manipulation of the laser tip, when a robot to perform operations on the gums is used. And in this article, it is the quality of the manipulations performed by the robot on typical trajectories identified in maxillofacial surgery, as compared with the surgeon, which is assessed. The quality of the manipulations primarily depends on the parameters of the process of cutting soft tissues, one of which is the air gap (or depth) between the tip of the laser and the soft tissues, which was analyzed in this article. Other parameters of the process by which the comparison is made, such as the accuracy of the trajectory and the speed of working it out are analyzed in the articles [15-18].

Conducting such a comparative study made it possible to quantitatively show how robotic manipulations will improve the quality of medical operations on the soft tissues of the oral cavity. From the point of view of scientific novelty, this study showed a quantitative relationship between a robot and a surgeon using the developed comparison criterion. The scientific usefulness of such a study makes it possible not only to apply modern robots (LBR 4+), but also gives direction to their improvement for the needs of maxillofacial surgery.

\section{Conclusion}

The results of the evaluation of the experiments show that by criterion - the average size of the air gap and by the additional criterion - the magnitude of the air gap spread on typical trajectories robot exceeds the natural abilities of the surgeon on average 7 times performing laser surgery in the maxillofacial region. 
Thus, the experiments carried out suggest that using the LBR $4+$ robot to perform functional movements with a diode laser tip allows you to maintain a more accurate air gap between the laser tip and biological tissue, compared to human (doctor's) arm movements, which allows for less invasiveness carrying out surgical operations.

\section{Funding}

Subsidy according to the state task of the Ministry of Health of the Russian Federation 056-00139-16

\section{Acknowledgments}

This article does not contain any studies with human participants or animals performed by any of the authors.

\section{Conflict of Interest}

The authors declare that they have no conflict of interest.

\section{Ethical approval}

This article does not contain any studies with human participants or animals performed by any of the authors.

\section{References}

1. Ballantyne GH. Robotic surgery, telerobotic surgery, telepresence and telementoring: review of early clinical results. Surg Endosc 2002; 16(10): 1389-1402. https://doi.org/10.1007/s00464-001-8283-7.

2. Kazanzides $\mathrm{P}$, Mittelstadt BD, Musits BL, Bargar WL, Zuhars JF, Williamson B, et al. An integrated system for cementless hip replacement. IEEE Eng Med Biol 1995; 14(3): 307-313. https://doi.org/10.1109/51.391772.

3. Goonewardene SS, Persad R. Robotic radical prostatectomy, day-case surgery and cardiac health: an opposing paradigm? J Robot Surg 2015; 9(4): 355. https://doi.org/10.1007/s11701-015-0537-9.

4. Vorotnikov AA, Poduraev YV, Romash EV. Estimation of error in determining the centers of rotation of links in a kinematic chain for industrial robot calibration techniques. Meas Tech 2015; 58(8): 864871. https://doi.org/10.1007/s11018-015-0809-9.

5. Zaret MM, Breinin GM, Schmidt H, Ripps H, Siegel IM, Solon LR. Ocular lesions produced by anoptical maser (laser). Science 1961; 134(3489): 1525-1526. https://doi.org/10.1126/science.134.3489.1525

6. Robustova TG, Bazikian ÉA, Ushakov AI, Daian AV, Serova NS, Ushakov AA. Combined clinico-roentgological approach in reconstructive surgery and maxillary sinus lifting for dental implantation. Russian Stomatology 2008; 1(1): 61-68. Russian. https://elibrary.ru/item.asp?id=23339914.

7. Genina EA. Biophotonic methods: phototherapy. Saratov, Russia: New Wind, 2012; 119 p. Russian. https://elibrary.ru/item.asp?id=25842269.

8. Chuniknin AA, Bazikyan EA, Pikhtin NA. A laser unit for photodynamic therapy and robot-assisted microsurgery in dentistry. Tech Phys Lett 2017; 43(6): 507-510. https://doi.org/10.1134/S1063785017060074.

9. Chunikhin AA, Sahakyan MY, Gazhva SI, Bazikyan EA. Development of nanosecond laser module built in the robotic multifunctional surgical complex for minimally invasive therapy of maxillofacial area pathology and estimation of its effects on blood plasma. Sovremennye tehnologii $\begin{array}{llll} & \text { medicine } & \text { 2016; } & \text { 30-35, }\end{array}$ https://doi.org/10.17691/stm2016.8.4.04.

10. Buynov MA, Bazikyan EA, Klimov DD, Poduraev YuV, Chunikhin AA. Surgical phantom. Russian Federation Patent for Utility Model No. 173509, claimed № 2017105725, dated 21.02.2017, publ. 29.08.2017. https://elibrary.ru/item.asp?id=38298115.
11. Shademan A, Decker RS, Opfermann JD, Leonard S, Krieger A, Kim PC. Supervised autonomous robotic soft tissue surgery. Sci Transl Med 2016; 8(337): 337ra64. https://doi.org/10.1126/scitrans/med.aad9398.

12. Jerbic B, Nikolic G, Chudy D, Svaco M, Sekoranja B. Robotic application in neurosurgery using intelligent visual and haptic interaction. Int $J$ Simul Model 2015; 14(1): 71-84. https://doi.org/10.2507/IJSIMM14(1)7.290.

13. Švaco $M$, Koren $P$, Jerbić $B$, Vidaković J, Šekoranja $B$, Šuligoj $F$. Validation of Three KUKA Agilus Robots for Application in Neurosurgery. In: Advances in Service and Industrial Robotics. RAAD 2017. Mechanisms and Machine Science. Springer: Cham, 2018; 49: 996-1006. https://doi.org/10.1007/978-3-319-61276-8 107.

14. Mönnich H, Stein D, Raczkowsky J, Wörn H. Increasing the accuracy with a rich sensor system for robotic laser osteotomy. In: The Eighth IEEE Sensors Conference Christchurch, 2009: 1684-1688. https://publikationen.bibliothek.kit.edu/1000031517.

15. Vorotnikov AA, Klimov DD, Romash EV, Bashevskaya OS, Poduraev YuV, Bazykyan EA, et al. Cutting velocity accuracy as a criterion for comparing robot trajectories and manual movements for medical industry. Mechanics \& Industry 2017; 18(7): 712. https://doi.org/10.1051/meca/2017047.

16. Vorotnikov AA, Klimov DD, Melnichenko EA, Poduraev YuV, Bazykyan EA. Criteria for Comparison of Robot Movement Trajectories and Manual Movements of a Doctor for Performing Maxillofacial Surgeries. Int J Mech Eng Robot Res 2018; 7(4): 361-366. https://doi.org/10.18178/ijmerr.7.4.361-366.

17. Vorotnikov AA, Buinov MA, Bushuev SV, Poduraev YuV, Chunihin AA Standard Deviation from the Average Cutting Velocity as a Criterion for Comparing Robot Trajectories and Manual Movements of a Doctor for Performing Surgical Operations in Maxillofacial Surgery. Int J Mech Eng Robot Res 2018; 7(3): 319-323. https://doi.org/10.18178/ijmerr.7.3.319-323.

18. Chunikhin AA, Poduraev YuV, Vorotnikov AA, Klimov DD, Sahakyan MY, Bazikyan EA. Efficiency Assessment of Nanosecond Laser Robotic Maxillofacial Area Surgery in Experiment. Sovremennye tehnologii v medicine 2017; 9(4): 123. https://doi.org/10.17691/stm2017.9.4.15.

\section{Authors:}

Andrey A. Chunikhin - MD, PhD, Associate Professor, Department of Oral Surgery, Moscow State University of Medicine and Dentistry n.a. A.I. Evdokimov, Moscow, Russia. http://orcid.org/0000-0002-9054-9464.

Ernest A. Bazikyan - MD, DSc, Professor, Head of the Department of Ora Surgery, Moscow State University of Medicine and Dentistry n.a. A.I. Evdokimov, Moscow, Russia. http://orcid.org/0000-0002-9184-3737.

Yury V. Poduraev - PhD, Professor, Head of the Department of Robotics and Mechatronics, Stankin Moscow State Technological University, Moscow, Russia. http://orcid.org/0000-0002-7585-6466.

Andrey A. Vorotnikov - Junior Researcher, Laboratory of Industrial Robotics, Mobile and Special Robotics, Mechatronic Modules and Digital Drives, Stankin Moscow State Technological University, Moscow, Russia. https://orcid.org/0000-0001-6371-8894.

Daniil D. Klimov - Junior Researcher, Laboratory of Industrial Robotics, Mobile and Special Robotics, Mechatronic Modules and Digital Drives, Stankin Moscow State Technological University, Moscow, Russia. https://orcid.org/0000-0001-6892-9324. 